\title{
Evaluation of the accuracy of Lachman and Anterior Drawer Tests with KT1000 in the follow-up of anterior cruciate ligament surgery
}

\author{
Bekir Eray Kilinc ${ }^{1, *}$, Adnan Kara ${ }^{2}$, Haluk Celik ${ }^{3}$, Yunus $0 c^{4}$, Savas Camur ${ }^{5}$ \\ ${ }^{1}$ Orthopaedics, State Hospital, Igdir, Turkey \\ ${ }^{2}$ Orthopaedics, Istanbul Medipol University, Istanbul, Turkey \\ ${ }^{3}$ Orthopaedics, State Hospital, Zonguldak, Turkey \\ ${ }^{4}$ Sisli Hamidiye Etfal Training and Research Hospital, Istanbul, Turkey \\ ${ }^{5}$ Orthopaedics, State Hospital, Catalca, Turkey
}

Ligament laxity measurement is clinically valuable to diagnose the injury and also to compare the laxity before and after surgical procedure. The aim of the study was to compare the accuracy of the Lachman and Anterior Drawer Tests to evaluate the knee examination with the KT1000 arthrometer after the anterior cruciate ligament (ACL) surgery in early follow-up period. Fourty ACL reconstructed knees were examined with the Lachman and Anterior Drawer Tests, and KT1000 arthrometer with compariable intact knee of the same patients. Physical emanination findings were compared with the KT1000 arthrometer with each power. Spearman correlation and receiver operating characteristic (ROC) analysis were used for the evaluation of relations between parameters. Significance was evaluated in $P<0.1$ and $P<0.05$. The mean age was $28.18 \pm 6.21 \mathrm{yr}$, and the mean follow-up was $23.09 \pm 9.08$ months The mean KT1000 measurements of 40 operated knees state at 6.8, 9.1,

\section{INTRODUCTION}

The anterior cruciate ligament (ACL) is the most frequently disrupted ligament in the human knee (Johnson, 1982). Measurements of the integrity of knee ligaments are used to diagnose injuries as well as to document the state of recovery. Ligament laxity measurement is clinically valuable to diagnosis injury and also to compare laxity before and after surgical procedure. However, few studies have examined the reliability of clinical methods or instruments used to measure anterior-posterior laxity. The Lachman test is characterized as the most direct and definitive evaluation examina-
$13.6 \mathrm{~kg}$ and maxium anterior displacement forces were $4.9,6.7,8.7$, and $11.9 \mathrm{~mm}$, respectively. The same values for the same acting forces of intact knees were 4.2, 5.9, 7.8, and 10.2, respectively. In Spearman's correlation and ROC analysis at 13.6-kg power on KT1000 arthrometer statistically matched with pyhsical examinations $(P<0.1, P<0.01)$. In our study, correlation of physical examinations with $\mathrm{KT} 1000$ arthrometer is a worthy evaluation technique that can be added to examination of $\mathrm{ACL}$ reconstructed knee to control with inexperinced examiners' findings. We suggest that at 13.6-kg power with KT1000 arthrometer findings perfectly match the Lachman and Anterior Drawer Tests of the knee.

Keywords: Lachman test, KT1000 arthrometer, Anterior Drawer Test, Anterior cruciate ligament, Anterior displacement, Knee examination
${ }^{*}$ Corresponding author: Bekir Eray Kilinc (ib http://orcid.org/0000-0003-1229-9815 Orthopaedics, State Hospital, Mehmer Akif Mah.23 Nisan Cad.No:66/2 Bucak/ Burdur 15300, Igdir, Turkey

Tel: +90-530-606-1884, Fax: +90-530-606-1884, E-mail :dreraykilinc@gmail.com Received: May 6, 2016 / Accepted: July 14, 2016 tion used to determine the status of ACL (Benjaminse et al., 2006; Cimino et al., 2010; Scholten et al., 2003). Anterior Drawer Test is also an accurate test for detecting ACL tear to evaluate anterior displacement of tibial manually a sensitivity of ACL tears (Calmbach and Hutchens, 2003). The KT1000 (MEDmetric Corp., San Diego, CA, USA) is a mechanical joint arthrometer allowing for stabilization of the femur with concurrent instrumented assessment of the lower leg (Daniel et al., 1985; Malcom et al., 1985).

The aim of the study was to compare the accuracy of the Lachman and Anterior Drawer Tests to evaluate the knee examination with the KT1000 arthrometer after surgery in early follow-up period. 


\section{MATERIALS AND METHODS}

Fourty patients were included the study. All patients signed a consent form after the test procedure was thoroughly explained. The patients had no reported history of re-injury in their involved knees. The patients included in the study who had minumum 6-month follow-up period and had the same surgery technique procedure. There was no involvement of the posterior cruciate ligament injury as detemined by a negative posterior sag test that was administered prior to each operation. All patients agreed via informed consent to participate in the investigation. The study was approved by the Institutional Review Board.

Fourty ACL reconstructed knees were examined with the Lachman and Anterior Drawer Tests and KT1000 arthrometer with compariable intact knee of same patients. Three examiners were included the study. One orthopaedic surgeon had 12-yr experience with evaluating knee stability and other had 9-yr experience. The licensed physical therapist had 25-yr experience with knee stability. The examinations were conducted with each examiner blind to the other's findings. After enrollment, each patient was brought to the room where the test were conducted. The active drawer test performed to the patient with knee flexed at $90^{\circ}$, hip at $45^{\circ}$ and foot stabilized on the firm examination table. While the foot was stabilized on the examination table and the hamstrings were relax, frequent manual gentle antero-posterior forces were applied to the proximal tibia, and tibia anteroposterior displacement in flexed knee was measured and recorded by the nurse based on International Knee Documentation Committee (IKDC) Knee Examination Form. The Lachman test was performed with the patient lying supine on a firm examination table and knee flexed to 20-30 degrees. The examiner's upper hand stabilized the unsupported distal thigh, while the lower hand, and finders feeling to ensure that the hamstring were relaxed, pulled the tibia forward. Anterior translation of tibia was measured and noted by same nurse based on IKDC Knee Examination Form. After the manual examination each patient was evaluated with the KT1000 arthrometer to record the milimiters of anterior translation at 6.8, $9.1,13.6 \mathrm{~kg}$ and the maximum power of force. The arthrometer attaches securely to the anterior leg with two straps. The arthrometer detects motion between two sensor pads, one in contact with the femur and the other in contact with the tibial tubercule. With a force-sensing handle, the examiner applies anterior and posterior displacement loads. The arthrometer emits an audible sound when $6.8,9.1,13.6 \mathrm{~kg}$ and maximum power force applied through the force handle. Two support platforms are necessary for the examination-a thigh support platform for positioning both knees of the patient in 20-30 degrees of flexion and a foot support platform platform for maintaining both feet 15 degrees from midline with the hips in external rotation. The examiners applied an anterior force by pulling slowly and smoothly on the force-sensing handle until four-consecutive audible tones indicated the each forces had been reached. Three trials of each measurement were completed. Measurements were noted by the nurse based on IKDC Knee Examination Form. Patients were instructed to relax between each trials. Arthrometer was removed and also placed on the contralateral knee. All examinations on one patient were performed by each of three examiners. For each patient the uninvolved knee was evaluated first in order to establish a baseline by which the contralateral knee could be utilizied. Every examiner followed the same examination procedure. Examiners paid attention to ensure the both knees were in the same degree of flexion during the physical examination procedure. All physical emanination findings were compared with the KT1000 arthrometer with each power.

PASS (Power Analysis and Sample Size) 2008 Statistical Software (NCSS, LLC, Kaysville, UT, USA) program was used for statistical analysis. Data were analyzed by using descriptive statistical methods (mean, standard deviation, median, frequency, ratio, minimum, maximum) and for comparing quantitative data Student $t$-test was used for three groups comparison of parameters with normal distribution, while Mann-Whitney $U$-test was used for three group comparisons of parameters without normal distribution. Spearman correlation analysis was used for the evaluation of relations between parameters. Significance was evaluated in $P<0.01$ and $P<0.05$.

\section{RESULTS}

The mean age of patients was $28.18 \pm 6.21 \mathrm{yr}$ (range, $17-40 \mathrm{yr}$ ), and the mean follow-up was 23.09 \pm 9.08 months (range, 9-42 months). Demographic information is presented in Table 1.

The mean KT1000 measurements of 40 operated knees state at 6.8, 9.1, $13.6 \mathrm{~kg}$ and maxium anterior displacement forces were 4.9, 6.7, 8.7, and $11.9 \mathrm{~mm}$, respectively. The same values for the

Table 1. Descriptive characteristics

\begin{tabular}{ll}
\hline Characteristic & Mean $\pm S D$ (range) \\
\hline Age (yr) & $28.18 \pm 6.21(17-40)$ \\
Follow-up (mo) & $23.09 \pm 9.08(9-42)$ \\
\hline
\end{tabular}

SD, stnadard deviaiton. 
Table 2. KT1000 measurement on operated and intact knees

\begin{tabular}{lcccc}
\hline & \multicolumn{4}{c}{ KT1000 } \\
\cline { 2 - 5 } & $6.8 \mathrm{~kg}$ & $9.1 \mathrm{~kg}$ & $13.6 \mathrm{~kg}$ & Max power \\
\hline Operated $(\mathrm{mm})$ & 4.9 & 6.7 & 8.7 & 11.9 \\
Intact $(\mathrm{mm})$ & 4.2 & 5.9 & 7.8 & 10.2 \\
\hline
\end{tabular}

The KT1000 (MEDmetric Corp., San Diego, CA, USA) is a mechanical joint arthrometer.

Table 3. KT1000 arthrometer comparison with Lachman and Anterior Drawer Tests $(n=40)$

\begin{tabular}{lcc}
\hline Differance operated and intact & Lachman & Anterior drawer \\
\hline $6.8 \mathrm{~kg}$ & & \\
$r$ & -0.117 & -0.158 \\
$P$-value & 0.471 & 0.331 \\
$9.1 \mathrm{~kg}$ & & \\
$r$ & -0.130 & 0.034 \\
$P$-value & 0.422 & 0.833 \\
$13.6 \mathrm{~kg}$ & & \\
$r$ & 0.367 & 0.430 \\
$P$-value & $0.02^{*}$ & $0.006^{* *}$ \\
Max power & & \\
$r$ & 0.024 & -0.191 \\
$P$-value & 0.882 & 0.238
\end{tabular}

The KT1000 (MEDmetric Corp., San Diego, CA, USA) is a mechanical joint arthrometer.

$r$, Spearman corelation ratio.

${ }^{*} P<0.05$. ${ }^{* *} P<0.01$.

same acting forces of nonoperated knees were 4.2, 5.9, 7.8, and 10.2 (Table 2).

All seven patients had ACL laxity with physical exams and also with the KT1000 arthrometer at 13.6-kg power. Those patients' findings were checked by magnetic resonance imaging. The results proved the ACL failure.

Spearman corelation test showed that there was a significant corelation between KT1000 arthrometer device at 13.6-kg power and Lachman and Anterior Drawer Tests $(P<0.1$ and $P<0.01$, respectively) (Table 3$)$. In receiver operating characteristic analysis at $13.6-\mathrm{kg}$ power on KT1000 arthrometer certainly matches with pyhsical laxity examination tests $(P<0.1)$ (Table 4, Fig. 1). Thirty pound on KT1000 arthrometer was the only one power that had statistically significancy.

\section{DISCUSSION}

After ACL rupture, most patients have detectable signs and symptoms of excess knee laxity and the joint becomes unstable. Anterior tibial translation in normal knees has very little different in right and left knees and in $95 \%$ of normal population; this dif-
Table 4. Receiver operating characteristic analysis of comparison of KT1000 arthorometer with Lachman and Anterior Drawer Tests

\begin{tabular}{lcccc}
\hline & \multicolumn{4}{c}{ Area under the curve } \\
\cline { 2 - 5 } & Area & SE & P-value & $95 \% \mathrm{Cl}$ \\
\hline KT1000 $(6.8 \mathrm{~kg})$ & 0.59 & 0.09 & 0.34 & $0.41-0.77$ \\
$\mathrm{KT} 1000(9.1 \mathrm{~kg})$ & 0.70 & 0.08 & $0.03^{*}$ & $0.53-0.86$ \\
$\mathrm{KT} 1000(13.6 \mathrm{~kg})$ & 0.66 & 0.09 & 0.07 & $0.50-0.83$ \\
KT1000 (maximum power) & 0.74 & 0.08 & $0.01^{*}$ & $0.58-0.90$ \\
Anterior Drawer & 0.54 & 0.09 & 0.65 & $0.36-0.72$ \\
Lachman & 0.54 & 0.09 & 0.63 & $0.36-0.73$
\end{tabular}

The KT1000 (MEDmetric Corp., San Diego, CA, USA) is a mechanical joint arthrometer.

$\mathrm{SE}$, standard error; $\mathrm{Cl}$, confidence interval.

${ }^{*} P<0.05$.

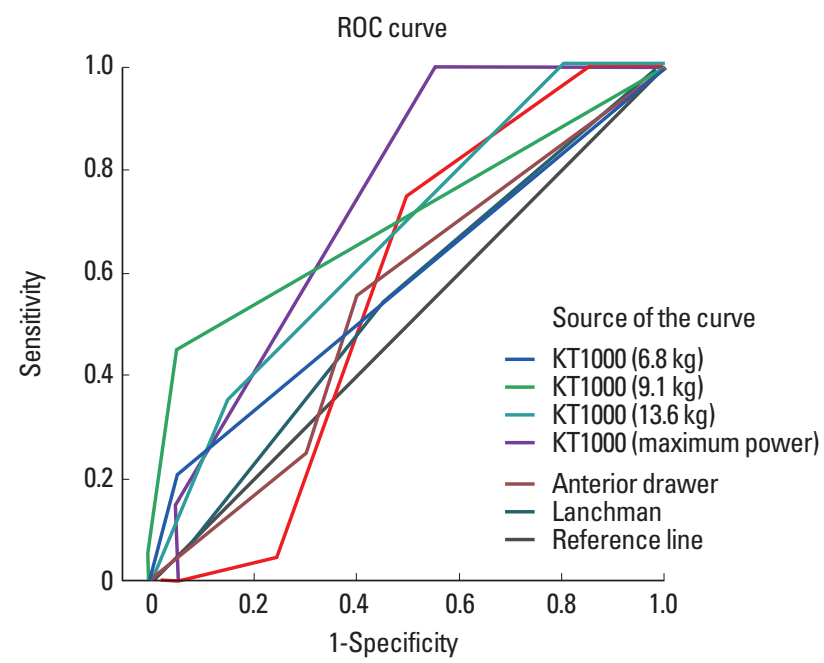

Fig. 1. Receiver operating characteristic (ROC) analysis of comparison of KT1000 arthorometer (MEDmetric Corp., San Diego, CA, USA) with Lachman and Anterior Drawer Tests.

ference is less than $2 \mathrm{~mm}$. It is crucial to use the contralateral normal knee as control to compare the difference in laxity between normal and injured knees (Shelbourne, 2010). In a clinical examination, the anterior displacement of the tibia on the femur is based on a subjective evaluation of ACL disruption or anterior instability. Several manual tests are available to determine whether the ACL has been injured and also to evaluate stability of ACL at follow-up period. The classical test for ACL deficiency, the Lachman and Anterior Drawer Tests are performed based on evaluation of the anterior instability (Solomon et al., 2001). Liu showed that the sensitivity of Lachman and Anterior Drawer Test was 95\% and $61 \%$, respectively. Lachman test sensitivity reaches up to 100\% under anesthesia (Liu et al., 1995). They are are the subjective evaluations most frequently used to assess ACL instability. 
Most commonly used devices available to assess ligament integrity are designed to assess the cruciate ligaments because they are accessible for testing and because they are important in maintaining knee stability. The Knee Arthrometer KT1000 is a commercially feasible instrument designed to provide and objective measurement of anterior-posterior laxity (Bach et al., 1990; Daniel et al., 1985; Daniel and Stone, 1990; Wroble et al., 1990). A study reported in 21 patients that KT1000 arthrometer can not be correlated with the clinical findings. They supported doubt on the accuracy and usefulness of the KT1000 (Graham et al., 1991).

More recently, testing of knee ligament integrity with devices such as the KT1000 arthrometer has gained increasing popularity. The KT1000 is a useful tool, as it is easy and fast to handle by an experienced examiner. Supplementary to noninvasive diagnostical methods it improves the confidence in clinical diagnosis. However, the importance of instrumental measurement should not be overrated even though reproducible results can be obtained. To compare the results of different studies the design has to be very similar.

It is important to know the accuracy of test used to establish a follow-up note in patients with knee stability. So far, there is no study has considered to correlate the physical examination test with the insrumented examination at follow-up period of patients who had ACL reconstruction surgery. In our study, physical tests' sensitivities certainly matched with the instrumented test KT1000 at 13.6-kg power. Therefore, the inexperienced examiner's interpretation could drop the sensitivity of these tests, regardless of which examiner findings could check with KT1000 instrumented examination. As for general practitioners who are less experienced in carying out these physical tests, they can certainly control their examination findings with the 30 pound power on KT1000 arthtrometer. This suggests that the reserved and equivocal physical test findings can be coralated with the KT1000 arthrometer after ACL surgery follow-up period.

With KT1000 instrument measurements are accurate in indicating anterior knee laxity, we tried to correlate the physical examinations of anterior-posterior laxity with KT1000 arthrometer device. Taken into consideration, our results report that the accuracy of KT1000 arthrometer device at 13.6-kg power was statistically correlated to the Lachman and Anterior Drawer Tests.

Based on the follow-up performance in our study, correlation of physical examinations with KT1000 arthrometer is a worthy evaluation technique that can be added to examination of ACL reconstructed knee to control with inexperinced examiners' findings. We suggest that at 13.6-kg power on KT1000 arthrometer findings perfectly match the Lachman and Anterior Drawer Tests of knee.

\section{CONFLICT OF INTEREST}

No potential conflict of interest relevant to this article was reported.

\section{REFERENCES}

Bach BR Jr, Warren RF, Flynn WM, Kroll M, Wickiewiecz TL. Arthrometric evaluation of knees that have a torn anterior cruciate ligament. J Bone Joint Surg Am 1990;72:1299-1306.

Benjaminse A, Gokeler A, van der Schans CP. Clinical diagnosis of an anterior cruciate ligament rupture: a meta-analysis. J Orthop Sports Phys Ther 2006;36:267-288.

Calmbach WL, Hutchens M. Evaluation of patients presenting with knee pain: Part I. History, physical examination, radiographs, and laboratory tests. Am Fam Physician 2003;68:907-912.

Cimino F, Volk BS, Setter D. Anterior cruciate ligament injury: diagnosis, management, and prevention. Am Fam Physician 2010;82:917-922.

Daniel DM, Stone ML. KT-1000 anterior-posterior displacement measurements. In: Daniel DM, Akeson WH, O'Connor JJ, editors. Knee ligaments structure, function, injury and repair. New York: Raven Press; 1990. p. 427-447.

Daniel DM, Stone ML, Sachs R, Malcom L. Instrumented measurement of anterior knee laxity in patients with acute anterior cruciate ligament disruption. Am J Sports Med 1985;13:401-407.

Graham GP, Johnson S, Dent CM, Fairclough JA. Comparison of clinical tests and the KT1000 in the diagnosis of anterior cruciate ligament rupture. Br J Sports Med 1991;25:96-97.

Johnson RJ. The anterior cruciate: a dilemma in sports medicine. Int J Sports Med 1982;3:71-79.

Liu SH, Osti L, Henry M, Bocchi L. The diagnosis of acute complete tears of the anterior cruciate ligament. Comparison of MRI, arthrometry and clinical examination. J Bone Joint Surg Br 1995;77:586-588.

Malcom LL, Daniel DM, Stone ML, Sachs R. The measurement of anterior knee laxity after ACL reconstructive surgery. Clin Orthop Relat Res 1985;(196):35-41.

Scholten RJ, Opstelten W, van der Plas CG, Bijl D, Deville WL, Bouter LM. Accuracy of physical diagnostic tests for assessing ruptures of the anterior cruciate ligament: a meta-analysis. J Fam Pract 2003;52:689694.

Shelbourne KD. The art of the knee examination: where has it gone? J Bone Joint Surg Am 2010;92:e9. 
Solomon DH, Simel DL, Bates DW, Katz JN, Schaffer JL. The rational clinical examination. Does this patient have a torn meniscus or ligament of the knee? Value of the physical examination. JAMA 2001;286:16101620.
Wroble RR, Van Ginkel LA, Grood ES, Noyes FR, Shaffer BL. Repeatability of the KT-1000 arthrometer in a normal population. Am J Sports Med 1990;18:396-399. 